\title{
Simulation Process for Mobile Nodes INFORMATION USING LOCATION-AIDED ROUTING IN MANET
}

\author{
Zarni Sann ${ }^{1}$ and Thi Thi Soe ${ }^{2}$ \\ ${ }^{1}$ Faculty of Computer System and Technology, Computer University (Mandalay), \\ Myanmar \\ ${ }^{2}$ Faculty of Computer Science, Computer University (Mandalay), Myanmar
}

\begin{abstract}
Location-aided routing protocol (LAR) utilizes the position information for routing. This system is simulated position information in specific areas by using mobile nodes. Movement of hosts results in routes, requiring some mechanism for determining new routes. This system finds position information using Location aided routing algorithm between each nodes. In real application, mobile nodes can apply without time consuming and saving cost to get location information using wireless sensor network. Each of the nodes is involved with calculating its position. If the position information is searched in the routing protocol, then position-based routing protocol is used. After creating the position table, all mobile nodes can transmit $64 B$ data within active mobile nodes via Base station. The algorithm is determined locations of nodes based on the connectivity for every node in specific area. This system is demonstrated location information by using Location-Aided Routing (LAR) protocol for route discovery. This system can assist to detect location of the specific areas using mobiles nodes. Simulation results are used to draw conclusions regarding to the proposed routing algorithm. The simulation of system is implemented mobile nodes and node movement function by using C\# programming language.
\end{abstract}

\section{KEYWORDS}

MANET, Location-Aided Routing (LAR), Position Information

\section{INTRODUCTION}

Location Aided Routing (LAR) uses directional forwarding flooding [3, 6, 7]. Infrastructure wireless networks, also known as cellular networks, have permanent base stations which are connected to other base stations through links. Mobile nodes communicate with another one through these base stations. Infrastructureless wireless networks, also known as ad hoc wireless networks, are a collection of wireless nodes that does not have any predefined Infrastructure or centralized control such as base stations or access points [9]. Location information may be provided by the Global Positioning System (GPS) in real implementation. With the availability of GPS, it is possible for a mobile host to know its physical location [1].

The objectives of this process are: to study Location Aided routing protocol in Mobile Ad hoc Network, to search node information in mobile network, to perform a restricted flooding in the region where the destination node, to reduce routing overhead, and mobile nodes can apply for searching position information with wireless sensor in many real world applications. This paper is organized into five parts including this section. Section 2 describes theoretical background that 
includes: Routing Protocol, Location-Aided Routing (LAR)and Position-based routing, etc. Section 3 discusses design and algorithm for LAR of the process. Section 4 explains the process for location information searching using location aided routing and creating position table for mobile node in specific area. Also this section includes the simulation result with figures. Finally, Section 5 presented conclusions and further extension items.

\section{Background Theory Of Routing Protocol}

In ad hoc networks, the routing protocols are divided into three categories: Proactive, Reactive and Hybrid. In Reactive routing protocols, the routes are discovered only when the source Mobile Node (MN) needs to transmit data packets. This system use reactive protocol $[5,6,7]$.

\subsection{Location-Aided Routing (LAR)}

When using LAR, any node needs to know its physical location [2, 5, 8, 9]. LAR is an on-demand source routing protocol. LAR uses location information for MNs to flood a route request packet for $\mathrm{D}$ in a forwarding zone instead of in the entire ad hoc network. The term forwarding zone in this paper is defined the same as the term request zone in [5].

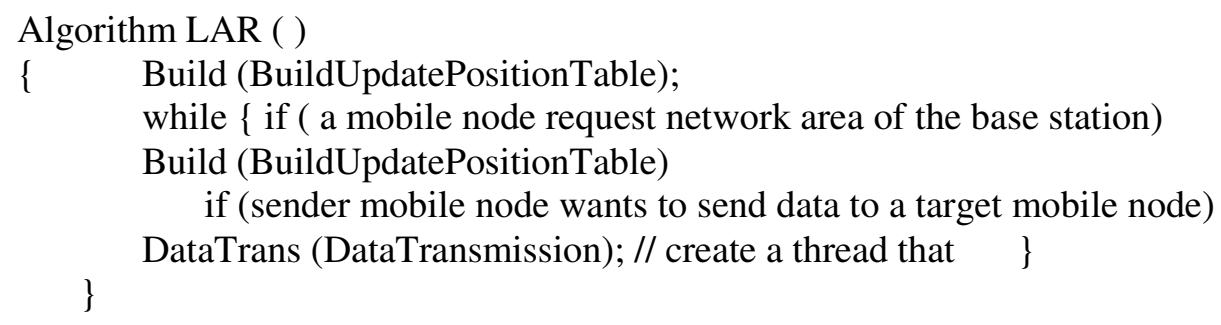

It creates a Build that executes BuildUpdatePositionTable which builds and updates the Position Table (PT) in Base Station (BS). Then, LAR executes an infinite loop. In this loop,BuildUpdatePositionTable procedure is called if a new mobile node enters the network area of BS. The new mobile node will contact its position to BS and hence, its position is entered in the PT in BS [2].

\subsection{Position-based Routing}

Position Table (PT) keeps the locations of all MNs using route packets. PT is created by Base Station through broadcasting small BEACON packets to all MNs in the network. In this paper, an $\mathrm{MN}$ uses a coordination of $(\mathrm{x}, \mathrm{y})$ position according to the mobile nodes locations. The positions of the two mobile nodes are (x1, y1) and (x2, y2) respectively. [4, 5, 6, 7].

\section{System Design AND ArChiteCtURE}

This simulation consists basically of two input files: mobile node File and Connection File. Mobile node File describes the mobile node ID for each node within the network. Connection File describes the traffic pattern and connections between nodes within the specific area. Connection Fileis generated in a completely random function which generates the mobile nodes and movement patterns. Position Table Creation Process is shown in figure 1.

Each node stored a Location Table, with entry information for every node within the specific area. This system will calculate distance and angle by using two equations. Base station send broadcast message to all mobile nodes in transmission range of hexagonal area. Then, the system 
create position table for each nodes. A location table entry for each node includes six fields as shown in figure 3.

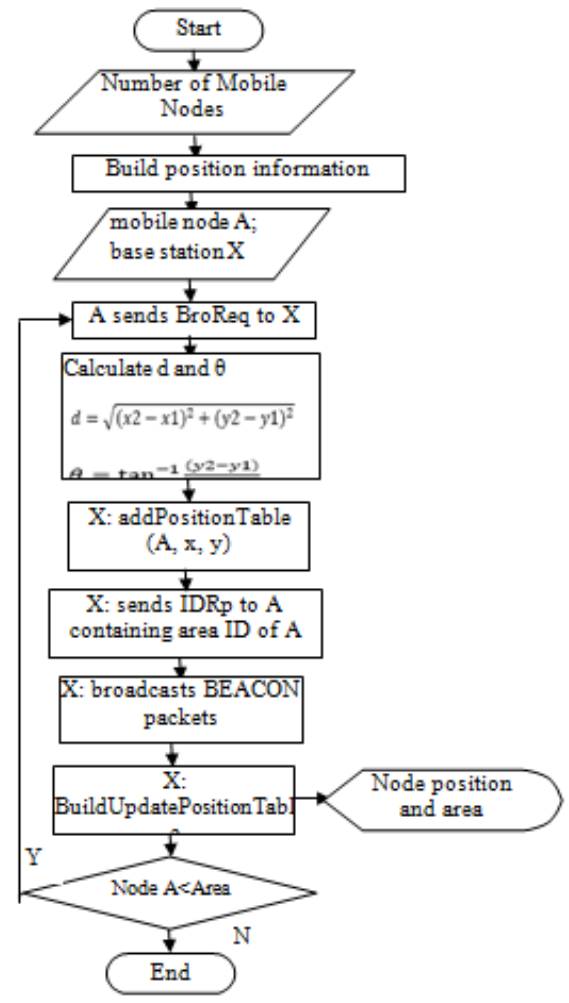

Figure 1. Position Table Creation Process

\subsection{Build Update Position Table Process}

When a mobile node A enters the network range of BS, node A uses its location estimation method to determine its ( $\mathrm{x}, \mathrm{y})$ position. A sends a broadcast message (BroReq message) that contains its position. BroReq message is a request to join the network of BS. BroReq contains the location of A. When BS receives all broadcast messages, it updates PT. BS determines N's angle $\theta$; distance $\mathrm{d}$ between node $\mathrm{N}$ and $\mathrm{BS}$; and classifies $\mathrm{N}$ as including in one of the six network areas $[2,5]$.

Procedure BuildUpdatePositionTable ( )

Input: mobile node $\mathrm{N}$; base station $\mathrm{BS}$;

\{ Control packet BroReq; // message containing x, y coordinates

if (node $\mathrm{N}$ enters network area controlled by X)

\{ $\mathrm{N}$ sends BroReq to BS; BS: addPositionTable ( N, x, y);

\}

BS: sends IDRp to $\mathrm{N}$ containing area no. of $\mathrm{N}$;

Repeat every Pause Time

base station BS sends BEACON message to node N; N sends BroReq to BS;

BS: UpdatePositionTable (N, $\mathrm{x}, \mathrm{y})$;

until valid timer expires

BS marks node A unreachable

\} 
Then, BS replies with ID Reply message (IDRp message) to A that contains the area ID of A, hence A knows its area ID. Then, BS periodically broadcasts BEACON packets to all MNs in the network in order to build PT that contains the network area ID of each MN that resides within the transmission range of BS. When a mobile node stops sending the broadcast packet (BroReq) then it is marked unreachable by BS after a timer T expires $[2,5]$. This system displayed the source nodes, destination nodes, up-to-date nodes, and out-of-date mobile nodes with different colors.

\subsection{Data Transmission Procedure}

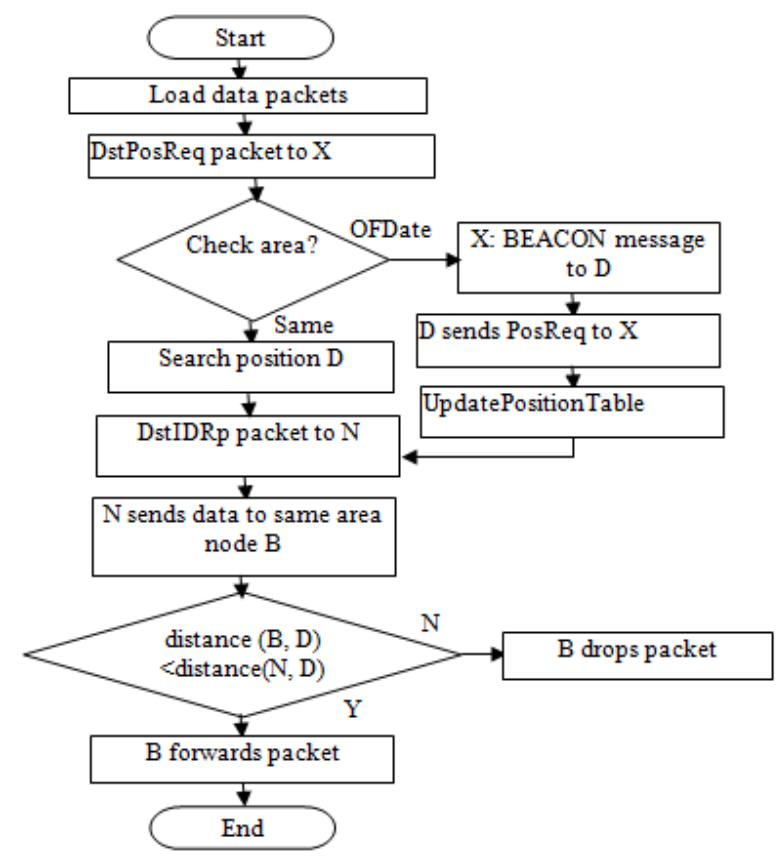

Figure 2.Data Transmission Flow Diagram

Data Transmission procedure is done when a source mobile node $\mathrm{N}$ sends data packets to a destination mobile node D. As Figure shows, first, $\mathrm{N}$ requests from BS to initiate a route discovery to node D by sending a DstPosReq (destination position request) packet to BS that requests the position information of $\mathrm{D}$. When $\mathrm{BS}$ determines the area ID of $\mathrm{D}$, it sends back DstIDRp (Destination ID Reply) packet to N containing the network area ID of D. Then, BS forwards data packets from node $\mathrm{N}$ to the area where $\mathrm{D}$ belongs only [2, 5].

This frees BS from being involved in the communication between $\mathrm{N}$ and $\mathrm{D}$ and BS will not be a performance bottleneck. If B's distance is less than the N's distance then B will forward the packet. Otherwise, it will drop it [2, 5]. Data Transmission process is shown in figure 2.

Procedure DataTransmission ( )

// Source (S) sends data to destination mobile node Destination (D)

Input: Data message, Sender node S, Receiver node D, base station X;

\{

$\mathrm{S}$ sends DstBroReq to X; X checks PT for position of D;

if (position of D in PT is out of date)

$\{\quad \mathrm{X}$ sends BEACON message to $\mathrm{D}$; 


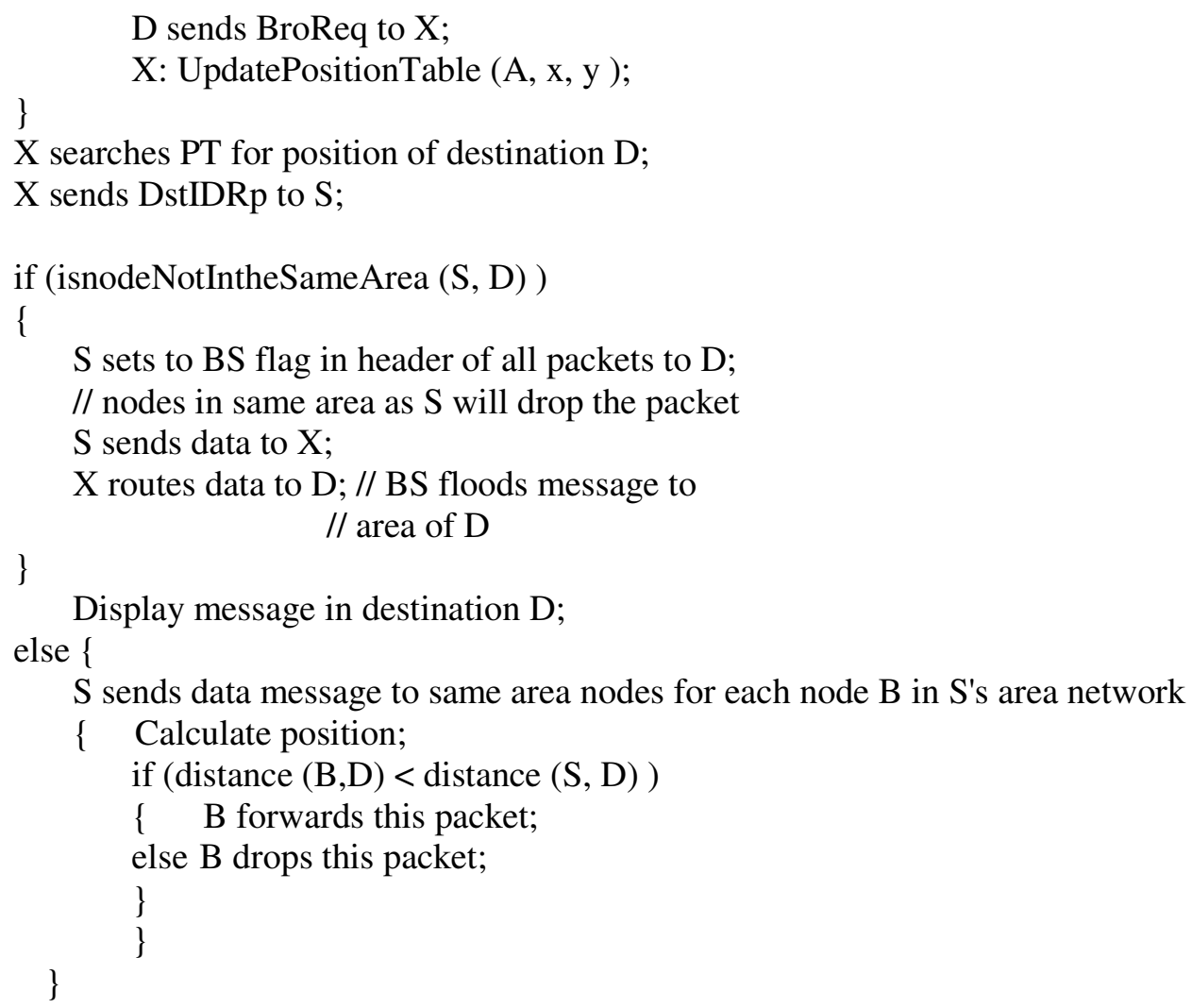

\section{Simulation Results Of The System}

This simulation process is demonstrated by using C\# programming language to get position information. In this simulation, it does not include the delays that may be introduced when multiple nodes attempt to transmit simultaneously. Transmission errors are also not considered. If a route reply is not received within a timeout interval, the sender uses the flooding algorithm to find the route. The timeout interval is a few seconds on average.

Number of nodes in the network was chosen to be between 50 and 100 for different simulation runs. The nodes in the network area are confined to a 1500 unit x 1500 unit hexagonal region. This system is used pause time to avoid movement faction during location information searching. This system considers average speeds (v) in the range 1.5 to 30.5 units/sec.Each node makes several movements during the simulation. A node does not pause between moves. For the simulation, a source node and a destination node are selected randomly. The source generates 4 data packets per second, with the time between two packets being exponentially distributed. Data can transmit within active region of six areas. If the small size of data transmits within the same region of different region, it will wait a small amount of time; data will arrive within a few second. If user sends the large amount of data in same or different region, it may be the large transmission time. This simulation is applied to search the specific area by using mobile nodes. If the nodes are not in the six area, it cannot search position information and the system must define "no information" (out-of-date mobiles) of this area. 


\subsection{Simulation Parameters}

- Simulation Area $: 1500 \mathrm{~m} \times 1500 \mathrm{~m}$

- Number of MNs : Total number of mobile nodes $($ minimum $=50$ and maximum $=100)$

- Transmission Range : 200m

- Movement Style : Random movement model

- Pause Time : The time in between direction changes for a node $(1,30,60 \mathrm{~s})$

- Data Payload : The size of each data packet (64 bytes)

- Packet Rate : 4 packet/s

\subsection{Simulation Results}

In figure 3, Base Station set in the center of network and creates the Position Table for up-to-date mobile nodes. Base Station(BS) contains the Position Table which consists of location of node, request time, situation and their network area ID. If the mobile node request it's position information, Base Station calculate the distance and angle of node to define the area ID in the network. Base station adds the node information and their situating areas ID at the position table. Position table keeps the information of nodes which are situated in the range of coverage area of the BS. This system simulates six areas of mobile nodes.

If source and destination node want to update the position information, these mobile nodes automatically send BEACON Message to Base Station. The mobile nodes in this simulation define Source nodes, Destination nodes, Base Station nodes, out-of-date and up-to-date nodes. Out-of-date nodes cannot send and receive the message because this node is not in the specific area. So the position table includes up- to-date mobile nodes information. The position table for mobile nodes information is shown in figure 3.

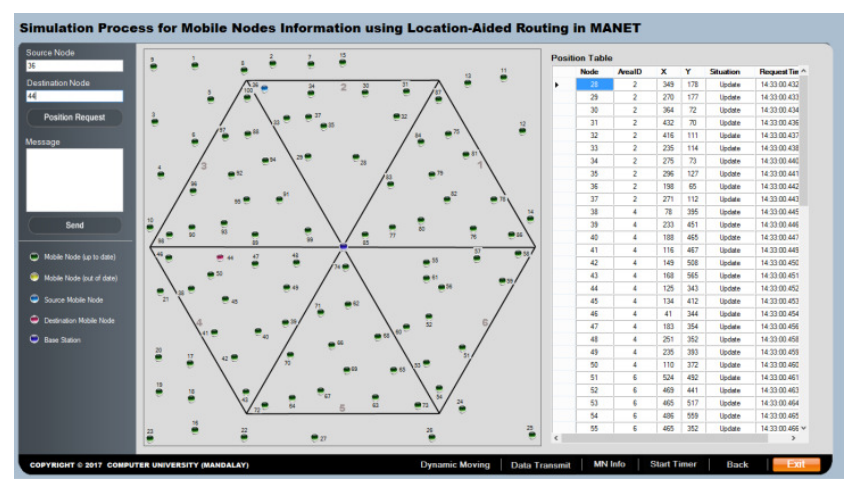

Figure 3. Position table creation process

Source and destination nodes are in the different areas, so source node must send message to Base Station at first. Then, Base Station find the position of destination node's information at Position Table and send the message belong to destination node' area as shown in figure 4 and figure 5 . 


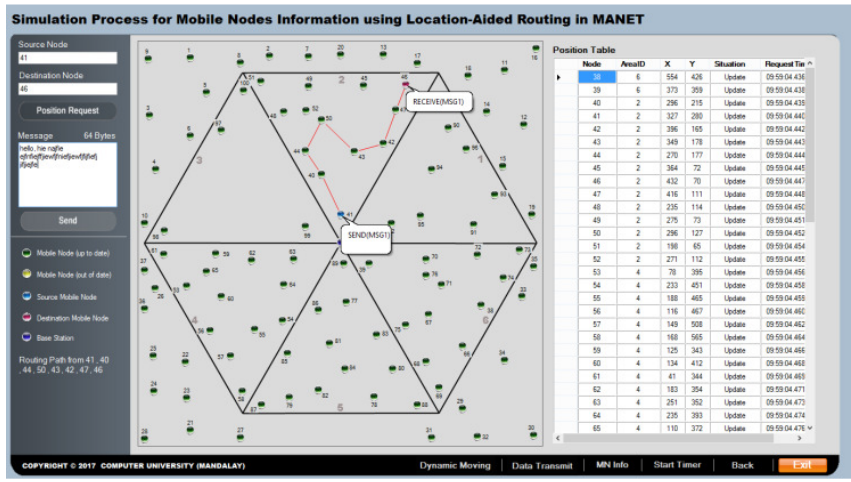

Figure 4.Sending Message from source node to destination node at same area

If sending node and receiving node are in the same area, Base station finds the position of receiver node at the Position Table and replies the sending message containing receiver 'position to sending node. Sender compare the distance between receiver node and other nodes in this area, then send the message to the less distance node information.

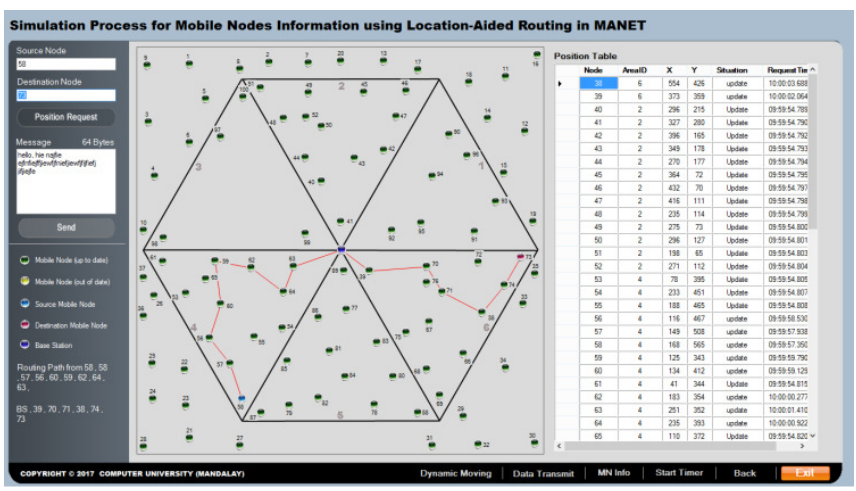

Figure 5.Sending Message from source node to destination node at different area

Finally, the message gets to the receiver node. This system defines the message with the size as 64 byte. If the nodes send messages over the 64 byte, system divides with $64 \mathrm{~B}$ and sends periodically to the destination node as shown in figure 6

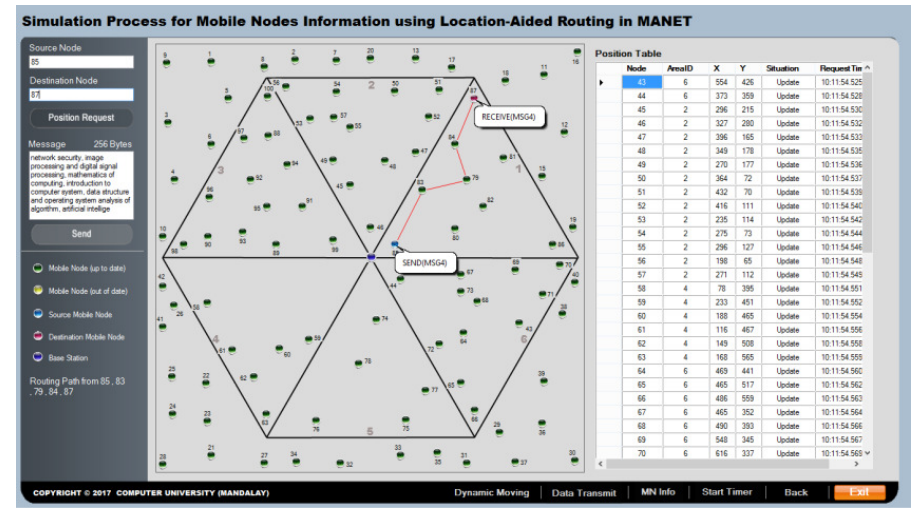

Figure 6. Multiple of $64 \mathrm{~B}$ data sending Message from source node to destination node 
When the destination node received message, the system displayed message sending time. Some nodes doesn't has in the specific area so, doesn't contain position information in the Position Table. These mobile nodes can't transmit and receive with other nodes, figure 7 display unreachable and list of mobile nodes in this simulation.

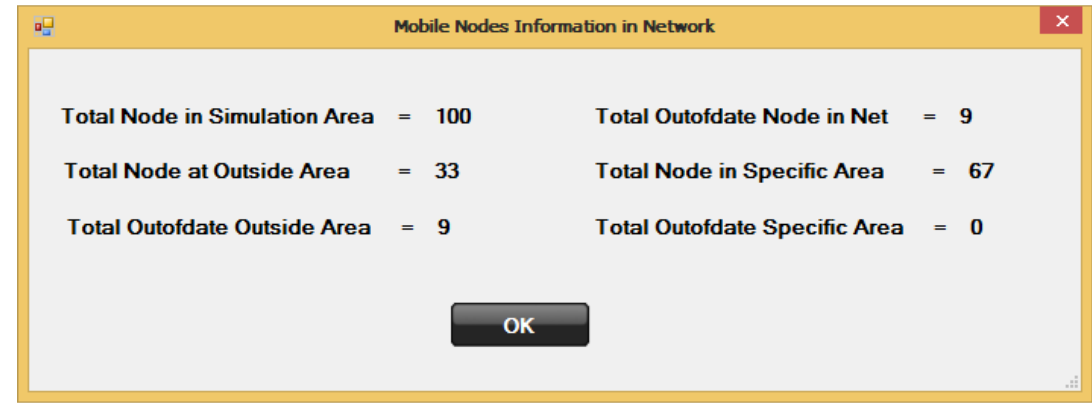

Figure 7.Number of Mobile Nodes in Network

All nodes move randomly in simulation area when the timer starts, so the position, situation and their area ID of each node may be changed. After changing process, this system can calculate the location of every node using BEACON message. This system is created again the position table after nodes changes as figure 8 .

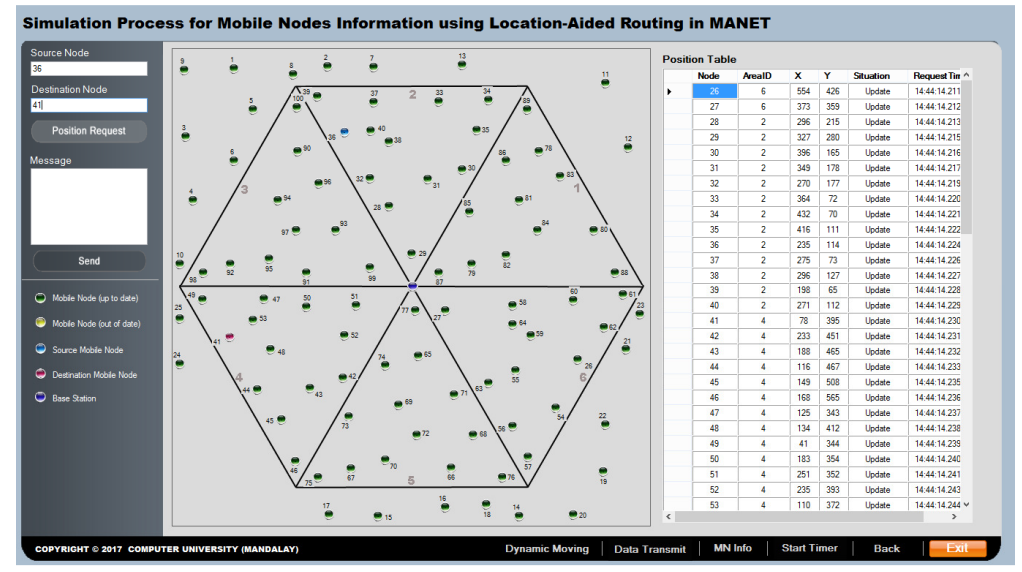

Figure 8. Mobile Nodes Dynamically changing process

This system can reduce processing overhead between Base station and every other node according to the position information. Every mobiles node can transmit messages over the 64 byte to each other, system divides message with $64 \mathrm{~B}$ and maximum message can transfer 256 Bytes. So transmission overhead can reduce over maximum packet in each node. All mobile nodes can transfer amount is $\mathrm{N}(\mathrm{N}-1) 4 . \mathrm{N}$ is number of mobile nodes and maximum packet can transmit (256 Bytes) 4 packets. So processing overhead and transmission overhead is as shown in Table 1 and 2. 
International Journal of Wireless \& Mobile Networks (IJWMN) Vol. 10, No. 2, April 2018

Table 1. Number of Nodes and Processing Overhead

\begin{tabular}{|c|c|c|c|}
\hline Mobile nodes & Outside Nodes & OutOfDateNodes & Processing Overhead(\%) \\
\hline 100 & 30 & 9 & 30 \\
\hline 70 & 25 & 5 & 36 \\
\hline 50 & 10 & 2 & 20 \\
\hline
\end{tabular}

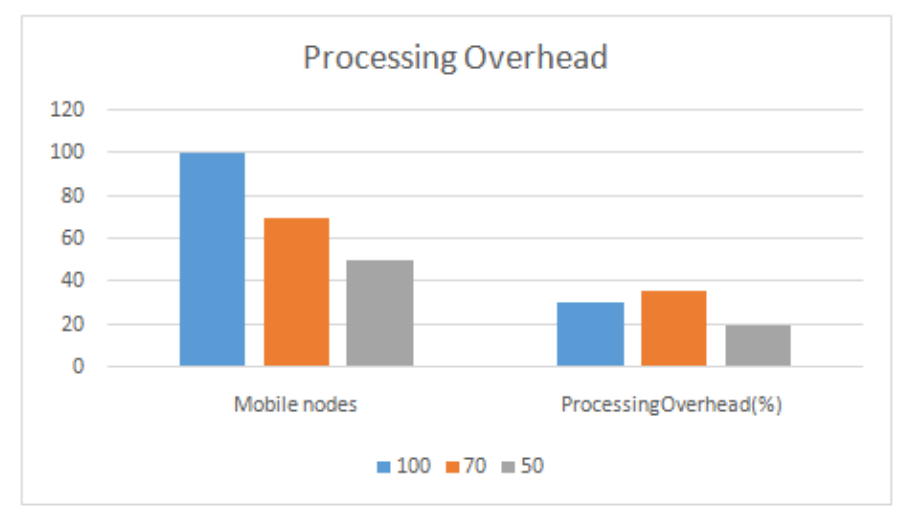

Table 2.Number of Nodes and Transmission Overhead

\begin{tabular}{|c|c|c|c|c|}
\hline Mobile nodes & Outside Nodes & Number of Packet & Transmitted Packet & TransmissionOverhead \\
\hline 100 & 30 & 39600 & 19320 & 20280 \\
\hline 70 & 25 & 19320 & 7920 & 11400 \\
\hline 50 & 10 & 9800 & 6240 & 3560 \\
\hline
\end{tabular}

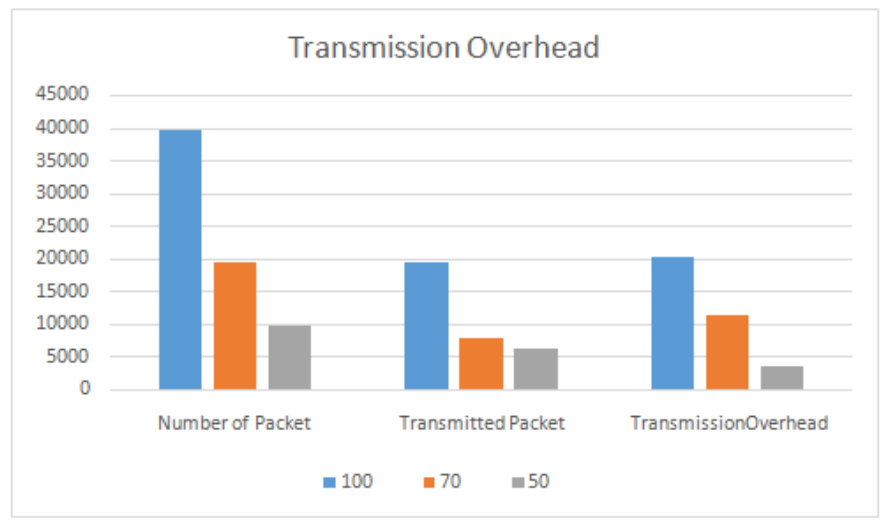

\section{Conclusion Of The System}

The approach of the network area into six sub-areas is not the optimal solution in all cases. Mobile node searching time is concerned with number of areas due to mobility. Based on this simulation, effective information can get by using wireless mobile nodes in specific range. If the organizations want to know important information about the specific area or difficulty environment, LAR algorithm support in wireless mobile network. Mobile nodes can move around 
Base Station for collecting information. By using Base station and dividing network area, routing information can get more quickly for each node. This system can experiment the transmission overhead and processing overhead according to total mobile nodes and position table.

This system can extend performance consideration for getting location information in different type of network patterns. In real world application, there may be speed, transmission impairment, out of range and out of date mobiles by using mobile nodes and random movement function. Further work is required to evaluate efficiency of these result, and also to develop other ways of using location information in ad hoc networks. In LAR, the search area is the fixed region containing both sender as well as receiver. If the real area may not be hexagonal region, it can be change the request and expect region.

\section{REFERENCES}

[1] Daniel C`amara Antonio A.F. Loureiro, "A Novel Routing Algorithm for Ad Hoc Networks", Proceedings of the 33rd Hawaii International Conference on System Sciences - 2000, 0-7695-0493$0 / 00 \$ 10.00$ (c) 2000 IEEE.

[2] Dr. P.K.Suri, Dr. M.K.Soni and ParulTomar, "Framework for Location Based Power Aware Routing in MANET", IJCSI International Journal of Computer Science Issues, Vol. 8, Issue 3, May 2011, ISSN (Online): 1694-0814, www.IJCSI.org.

[3] HimadriNathSaha, Dr. Debika Bhattacharyya, Dr. P. K.Banerjee, "A novel and Efficient Secured Routing Algorithm in Mobile Ad hoc Network", DOI : 10.5121/ijwmn.2011.3519, International Journal of Wireless \& Mobile Networks (IJWMN) Vol. 3, No. 5, October 2011.

[4] Johnson D, Maltz D. "Dynamic Source Routing in ad hoc wireless networks. Mobile Computing". (edited by Imielinski T, Korth H); Kluwer Academic, 153-181, 1996.

[5] Mohammad A. Mikki, "Energy Efficient Location Aided Routing Protocol for Wireless MANETs", (IJCSIS) International Journal of Computer Science and Information Security, Vol. 4, No. 1 \& 2, 2009

[6] Mamoun Hussein Mamoun, "Location Aided Hybrid Routing Algorithm for MANET", International Journal of Engineering \& Technology IJET-IJENS Vol: 11 No: 01, 111901-3838 IJET-IJENS @) February 2011.

[7] Sanjay Singh, AkshataTrivedi, Dr. Sanjeev Sharma, "A Modified Location Aware Routing Algorithm for MANET”, International Journal of Software and Web Sciences (IJSWS), ISSN (Print): 2279-0063 ISSN (Online): 2279-0071, 2013.

[8] Tzay-Farn Shih, Hsu-Chun Yen, "Location-aware routing protocol with dynamic adaptation of request zone for mobile ad hoc networks", Springer Wireless Network 14:321-333,DOI 10.1007/s11276-006-9955-y, 2008.

[9] Y. Ko and N. H. Vaidya. "Location-aided routing (LAR) in mobile ad hoc networks", ACM Wireless Networks, Vol. 6, No. 4, pp. 307-321, July2000. 
International Journal of Wireless \& Mobile Networks (IJWMN) Vol. 10, No. 2, April 2018

\section{Authors}

ZarniSann is currently working in Faculty of Computer System and Technology at Computer University (Mandalay). She received B.Sc. Degree in Physics from Mandalay University in 1996. She obtained D.C.Sc and M.I.Sc in Information Technology from University of Computer Studies, Mandalay and Yangon in 1998 and 2000 respectively. In May 2004, she completed the Ph.D. degrees in Information Technology from the University of Computer Studies, Yangon (UCSM). She has received four International Conference papers. She has supervised about 60 Master students in IT field from 2007 to 2013. She's current research interests include Network Security, Cloud computing and Wireless and Mobile Network.

ThiThiSoe is currently working in Faculty of Computer Science at Computer University (Mandalay). She received B.Sc. Degree in Physics from Mandalay University in 1996. She obtained D.C.Sc and M.I.Sc in Information Technology from University of Computer Studies, Mandalay and Yangon in 1998 and 2000 respectively. In 2007, she completed the Ph.D degrees in Information Technology from the University of Computer Studies, Yangon (UCSM). She has received five International Conference papers. She has supervised about 60 Master students in IT field from 2008 to 2013. She's current research interests include Image Processing, Signal Processing and Wireless and Mobile Network
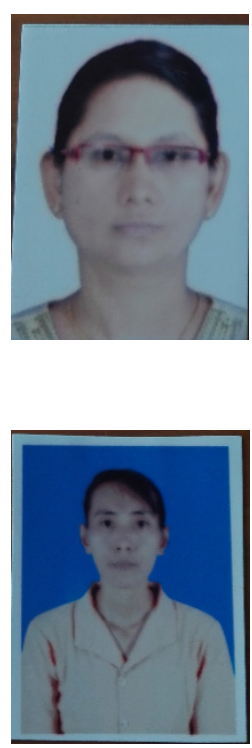